\section{Kind verstaucht sich den Knöchel: Die Wachstumsfuge bleibt meist heil}

Soll man Kinder mit verletztem Knöchel und Verdacht auf eine Beteiligung der fibulären Epiphysenfuge mit einem Gipsverband immobilisieren? Die Ergebnisse einer kanadischen Studie sprechen dagegen. Den Autoren zufolge liegen den Schmerzen und Schwellungen auch bei Kindern in den meisten Fällen Bandverletzungen zugrunde.
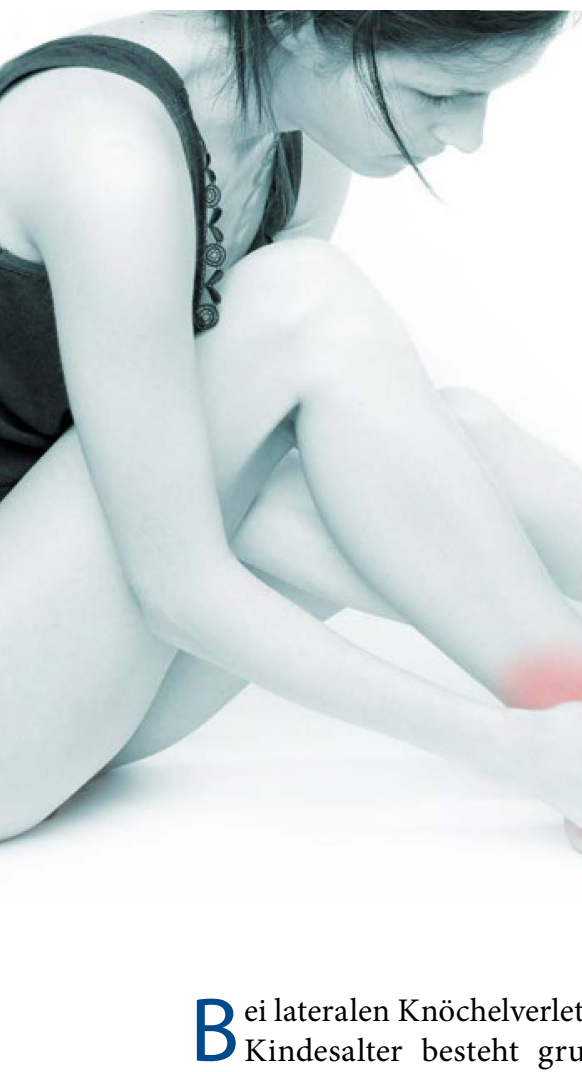

Ein seitliches Umknick-Trauma führt auch bei Kindern überwiegend zu Bandläsionen und nicht zu Frakturen.

B i lateralen Knöchelverletzungen im Kindesalter besteht grundsätzlich die Sorge, die Wachstumsfuge der Fibula könnte beteiligt sein. Standard-Röntgenaufnahmen des Knöchels in drei Ebenen können eine nicht dislozierte Fraktur der distalen Fibula vom Typ Salter-Harris I (SH1DF) nicht sicher ausschließen. Um Wachstumsstörungen vorzubeugen, wird daher oft schon beim Verdacht auf eine solche Verletzung trotz negativen Röntgenbefunds eine drei- bis sechswöchige Immobilisierung mittels Gips oder Schiene propagiert.

Gegen dieses Vorgehen wenden sich nun Kathy Boutis und Kollegen, Universität Toronto. In ihrer MRT-Studie zeigte das Team, dass sich der Verdacht einer SH1DF nur in 3\% der Fälle bestätigt. Die Schlüsselbotschaft: Auf 33 Kinder, die mittels Gips immobilisiert werden, kommt nur eine SH1DF. Für das funktionelle Ergebnis bringt die Immobilisierung offenbar keinen Vorteil.

An der prospektiven Kohortenstudie waren 135 Kinder im Alter zwischen fünf und zwölf Jahren beteiligt. Bei allen war der laterale Knöchel über der distalen Wachstumsfuge der Fibula nach einer akuten Verletzung geschwollen oder schmerzhaft. Der Röntgenbefund war in allen Fällen negativ ausgefallen. Um die klinisch gestellte Verdachtsdiagnose $\mathrm{SH}$ 1DF auszuschließen, wurden die Kinder innerhalb von einer Woche nach dem Unfall einer MRT-Untersuchung unterzogen. Behandelt wurden sämtliche $\mathrm{Pa}$ tienten mit einer abnehmbaren Schiene; Bewegung war erlaubt, sofern sie von den Kindern toleriert wurde.

Nur in vier Fällen bestätigte die MRT den Verdacht einer SH1DF; bei zweien davon zog sich die Verletzung (abnormes Signal in der T2-gewichteten Aufnahme) durch die gesamte Wachstumsfuge, die restlichen zwei hatten eine Verletzung, die sich nur über maximal die Hälfte der Epiphysenfuge erstreckte. 38 Kinder wiesen im MRT-Bild eine Ausrissfraktur der Fibula auf. Eine Verletzung der Bänder fand sich bei $80 \%$ der Patienten. Zu 67,6\% handelte es sich um mittel- bis hochgradige Verletzungen.

Wie die Autoren berichten, war der Verlauf sowohl mit als auch ohne SH1DFBefund im MRT exzellent: Nach einem Monat konnten 72,1\% der Kinder ohne Probleme voll belasten, nach drei Monaten waren es 96,1\%. Auf der „Activity Scale for Kids" lagen die Kinder mit MRT-bestätigter distaler Fibulafraktur nach einem Monat bei 82,0 \% (Optimum: $100 \%$ ), jene ohne Fraktur bei 85,8 \% - ein nicht signifikanter Unterschied von 3,8\%.

Am Ende der dreimonatigen Nachbeobachtung standen die Daten von 37 Kindern mit MRT-bestätigter Fraktur jeglicher Art zur Auswertung zur Verfügung. Von diesen waren alle in der Lage, ihre früheren Aktivitäten auszuführen. Von den 91 Kindern ohne Fraktur waren es $95,6 \%$.

Fazit: Die Studie hat nach Meinung der Autoren bestätigt, dass bei Kindern nach einem Knöcheltrauma mit negativem Röntgenbefund nur selten mit einer Beteiligung der fibulären Wachstumsfuge zu rechnen ist. Selbst wenn der Verdacht auf eine SH1DF zutreffe, sei die Behandlung mit einer abnehmbaren Schiene offenbar ausreichend und eine mehrwöchige Immobilisierung im Gips unnötig.

Ob die Kinder langfristig von Wachstumsverzögerungen verschont bleiben, müssen weitere Studien mit längerer Laufzeit zeigen. Nach der bisherigen Datenlage seien solche Störungen bei nicht dislozierten Frakturen der distalen Fibula aber äußerst selten, betonen die Autoren. Schließlich finde das Längenwachstum vornehmlich in der proximalen Wachstumsfuge statt.

Dr. Elke Oberhofer

Boutis K et al. Radiograph-Negative Lateral Ankle Injuries in Children: Occult Growth Plate Fracture or Sprain? JAMA Pediatr 2016; 170(1): e154114; doi: 10.1001/jamapediatrics.2015.4114. 